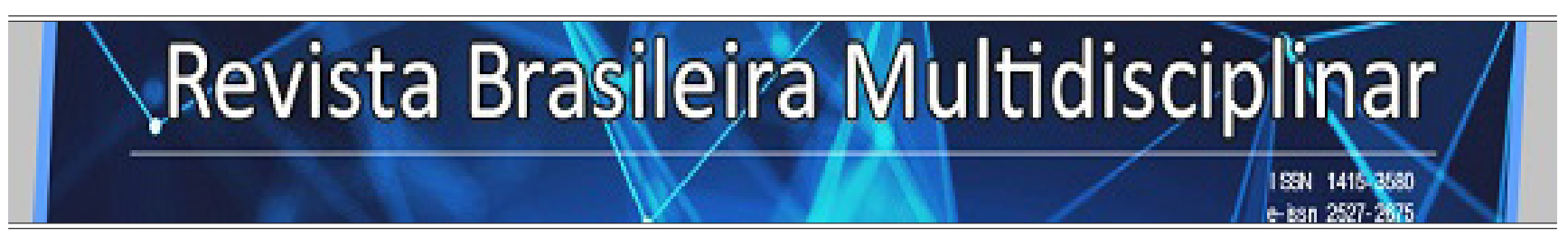

http://revistarebram.com/index.php/revistauniara

\title{
Rastreamento de Sistomas de depressão em POliciais Penais: Estudo de Validação do PHQ-9
}

Rodrigo de Souza ${ }^{\star}$ Fabio Biasotto Feitosa ${ }^{\star *}$; Tomás Dabiel Menéndez Rodríguez ${ }^{\star * *}$; Leandro Aparecido Fonseca Missiatto ${ }^{\star * \star *}$.

* Mestre pelo Programa Mestrado Acadêmico em Psicologia da Universidade Federal de Rondônia (UNIR).

${ }_{*}^{*}$ Doutorado em comportamento, desenvolvimento cognitivo e saúde. Universidade Técnica de Lisboa/UTL .

*** Doutorado em Matemática na Saint Petersburg State University.

${ }_{* * * *}$ Mestrado em Psicologia na Universidade Federal de Rondônia - UNIR.

*Autor para correspondência e-mail: rodrigopsibr@gmail.com

\section{Palavras-chave}

Cicatrização de Feridas

Depressão

Validação

Polícial Penal

\section{KEYWORDS}

Depression

Validation

Prison Officer

\begin{abstract}
RESUMO
A depressão é um grave transtorno psicológico que acomete cada vez mais pessoas, contudo, seu diagnóstico precoce necessita de instrumentos eficazes que possam rastrear suas sintomatologias em diversificadas bases populacionais. O objetivo deste estudo foi o de contribuir com o corrente processo de validação do questionário PHQ-9 para o uso na atenção primária em saúde pública no rastreio de sintomas depressivos. Trata-se de uma pesquisa de levantamento realizada com uma amostra de 131 policiais penais lotados no Presídio Federal de Porto Velho/RO. Utilizou-se como instrumentos de pesquisa o Patient Health Questionnaire-9 (PHQ-9), a Escala de Hamilton (HAM-D) que serviu como regra de ouro para a verificação da eficácia do PHQ-9 no rastreio de sintomas depressivos, além de um questionário sociodemográfico para identificação do perfil da amostra. Os resultados demonstraram forte e significativa correlação ente as escalas (rho = 0,667; $\mathrm{p}<0,001$ ) o que sugere a confiabilidade do PHQ-9 para rastreio de sintomas depressivos. Conclui-se que o PHQ-9 pode ser utilizado como instrumento confiável na rede de saúde pública por profissionais qualificados, principalmente os alocados na atenção primária, auxiliando-os no diagnóstico precoce da depressão.
\end{abstract}

TRACKING DEPRESSION SYMPTOMS IN FEDERAL PRISION OFFICERS: PHQ-9 VALIDATION STUDY Depression is a serious psychological disorder that affects more and more people, and for its early diagnosis it is necessary to have effective instruments that can track its symptoms in diverse population bases. The aim of this study was to contribute to the current PHQ-9 questionnaire validation process for use in primary public health care to screen for depressive symptoms. This is a survey carried out with a sample of 131 prison officers assigned to the the Federal Prison of Porto Velho/RO. The Patient Health Questionnaire-9 (PHQ-9), the Hamilton Scale (HAM-D), which served as the golden rule for verifying the effectiveness of PHQ-9 in screening for depressive symptoms, were used as research instruments, plus a sociodemographic questionnaire to identify the sample profile. The results demonstrated a strong and significant correlation between the scales (rho $=0.667 ; \mathrm{p}<0.001$ ), which suggests the reliability of the PHQ-9 for screening for depressive symptoms. It is concluded that PHQ-9 can be used as a reliable instrument in the public health network by qualified professionals, especially those allocated to primary care, helping them in the early diagnosis of depression. 


\section{INTRODUÇÃo}

A depressão é um transtorno do humor caracterizado por uma experiência subjetiva de grande sofrimento, que acomete o indivíduo com diversas perdas tanto orgânicas quanto psicossociais podendo levar até à ideação suicida. Essas alterações podem interferir no comprometimento do desempenho psicossocial no trabalho, vida acadêmica e relações interpessoais (MISSIATTO; FEITOSA; RODRÍGUEZ, 2019). Segundo a Organização Pan-Americana da Saúde (OPAS), estima-se que atualmente mais de 300 milhões de pessoas em diversas idades sofram com esse transtorno psicológico (OPAS, 2020).

Estima-se também que 23,9\% dos casos psiquiátricos atendidos na atenção primária de saúde seja de depressão (MOLINA et al., 2012), todavia este número está longe de representar a realidade, uma vez que muitas pessoas com depressão podem passar pela atenção básica de saúde sem o devido diagnóstico (ARANTES, 2007).

O diagnóstico é importante para que o paciente tenha acesso o quanto antes a terapêutica adequada. Geralmente esse diagnóstico é realizado por entrevistas que evidenciam a história clínica do paciente, tempo de evolução dos sinais e sintomas apresentados, além de aplicação de escalas específica (LOSSNITZER et al., 2013; GUERRA et al., 2018). Estudos de levantamento sobre instrumentos para rastreio de sintomas de depressão demostram existir uma variabilidade grande, como a pesquisa realizada por Aros e Yoshida (2009), que identificaram 49 escalas para detectar sintomas depressivos.

Essa diversidade de instrumentos é favorável por possibilitar aos profissionais de saúde uma vasta gama de opções técnicas para chegar ao diagnóstico da depressão. Contudo, é preciso que tais instrumentos tenham passado por estudos internacionais e nacionais para validação de sua capacidade de rastreio e diagnóstico de depressão, propiciando aos profissionais de saúde segurança clínica para as avaliações e definição de estratégias de intervenção.

Nesse sentido, algumas pesquisas vêm sendo conduzidas para validação de instrumentos que auxiliem no diagnóstico da depressão, dentre essas se destacam as pesquisas que buscam validar no Brasil o Patient Health Questionnaire ( $P H Q-9$ ), instrumento que permite um rápido e adequado rastreamento de sintomas depressivos em adultos. Ele é amplamente utilizado no mundo devido a sua fácil aplicação e resultados fidedignos para identificação de sintomatologia depressiva.

Em estudo realizado por Santos et al. (2013), cujo objetivo foi o de estudar a validade do PHQ-9 no rastreio de episódio depressivo maior na população geral, aplicou-se o instrumento em uma amostra diversificada de 447 pessoas da cidade de Pelotas/RS. Os resultados identificaram o ponto de corte $\geq 9$ como de máxima sensibilidade $(77,5 \%)$ e especificidade (86,7\%). A pesquisa ainda utilizou de análises algorítmicas para verificar a sensibilidade do instrumento para depressão maior, nesse caso houve diminuição da sensibilidade para 42,5\%, enquanto que a especificidade aumentou para 95,3\%. O PHQ-9 mostrou-se apropriado para o rastreamento de episódio depressivo maior, sendo mais eficaz a pontuação contínua do que a algorítmica para rastreamento de episódio depressivo maior. Contudo, os pesquisadores alertam cautela para se utilizar a nota de corte para diagnóstico, sendo necessários estudos específicos com outras bases populacionais para que as peculiaridades de cada localidade sejam respeitadas.

Na pesquisa conduzida por Guerra et al. (2018), em que se buscou verificar a concordância entre os métodos de rastreamento para depressão em pacientes com insuficiência cardíaca através dos PHQ-9, Escala de Avaliação de Depressão de Hamilton (HAM-D) e Inventário de Depressão de Beck-II (BDI-II), obteve-se concordância diagnóstica entre os três instrumentos de apenas 47,4\% com p =0,000. Tais resultados sustentam a relativa qualidade do PHQ-9 no rastreamento para sintomas depressivos na amostra estuda, contudo, os autores observaram alto percentual de falsos negativos, justificando o investimento em mais pesquisas de rastreamento concomitante com outros instrumentos.

Em um recente estudo realizado por Zimmerman (2019) que discute o uso do PHQ-9 com pacientes com depressão, utilizou-se desse instrumento como escala para definir o tipo e a duração da intervenção 
clínica contra a depressão, mostrando-se mais eficaz quando comparado com tratamentos efetuados a partir de critérios diagnósticos convencionais, com taxa de resposta significativamente maior $(67,0 \% \mathrm{x}$ $59,7 \%$ ), além de acentuada taxa de remissão (46,7\% x 42,8\%) para depressão. Esses dados demonstram a importância desse instrumento para identificação de sintomas depressivos na atenção primária de saúde.

Notam-se diversificados estudos bem-sucedidos no que concerne à validação do PHQ-9 para rastreio de sintomas depressivos, tais pesquisas quando realizadas com distintas bases populacionais amplia a segurança para o uso desse instrumento na atenção primária de saúde, destacando-se como um importante aliado para triagem e definição de acompanhamento adequado no tratamento de depressão. A população utilizada para composição da amostra deste estudo no rastreio de depressão foi uma amostra de policiais penais da cidade de Porto Velho, capital do estado de Rondônia.

Levou-se em consideração a escassez de pesquisas realizadas com esses servidores públicos quanto à manifestação de sintomas depressivos, bem como o reconhecimento na literatura de que esse contingente populacional, em virtude das peculiaridades de seu trabalho, está mais sujeito ao adoecimento mental (FERNANDES et al., 2002; MORAES, 2013; WEBB-MURPHY et al., 2015; BEZERRA et al., 2016). Além disso, a escolha da população estudada deveu-se ao fato de ainda não haver estudos de validade do PHQ9 com policiais penais, mesmo sendo uma categoria altamente vulnerável ao adoecimento psíquico em virtude de seu trabalho.

Em vista de colaborar com esses esforços, o objetivo deste estudo foi o de contribuir com o corrente processo de validação do questionário PHQ-9 para o uso na atenção primária em saúde pública no rastreio de sintomas depressivos.

\section{MATERIAIS E MÉTODO}

A pesquisa foi aprovada pelo Comitê de Ética em Pesquisa da Universidade Federal de Rondônia (CAAE: 44394615.7.0000.5300). Todos os cuidados éticos determinados pelas diretrizes de pesquisa com seres humanos foram devidamente observados. A coleta de dados ocorreu dentro do Presídio Federal de Porto Velho/RO com aplicação dos instrumentos de modo individual. A amostra foi composta por 131 (cento e trinta e um) servidores de um total de 140, o que proporcionou à pesquisa um nível de confiança de $95 \%$ com margem de erro de 3,6.

Como instrumento de pesquisa utilizou-se:

Escala de Hamilton-D (HAM-D): Este instrumento foi usado como padrão ouro para comparação com o PHQ-9. A Escala de Hamilton verifica os níveis de depressão do indivíduo testado. Os escores vão da normalidade até a depressão muito grave. A escala tem o propósito de quantificar os sintomas e sua gravidade. Possui 21 (vinte um) subitens na versão completa, que possuem entre três e cinco respostas possíveis. A pontuação máxima pode chegar a 62 (sessenta e dois) na versão completa. O resultado de cada item é determinado de 0 a 2, ou de 0 a 4, de acordo com o número de opções em cada subitem. Para definir o escore, usam-se os seguintes cortes: não deprimido, de 0 a 7; pouco deprimido, de 8 a 13; depressão moderada, de 14 a 18; depressão grave, 19 a 23; e muito grave, acima de 23; estudos apresentaram consistência interna e homogeneidade nos seus construtos (coeficientes alfa entre 0,66 e 0,78) (FREIRE et al., 2014).

Questionário sobre a Saúde do Paciente - 9 (PHQ-9): O PHQ-9 foi criado com o objetivo de rastrear sinais e sintomas da depressão maior, determinando o grau de sua severidade (OSÓRIO et al., 2009). Seu foco são as duas últimas semanas e possui nove itens que tratam acerca de problemas característicos da depressão em uma escala de quatro pontos ( $0=$ nenhuma vez, $3=$ quase todos os dias) relacionados à frequência com que foram percebidos pelo respondente. Ao final há uma pergunta que verifica de que maneira os sintomas afetaram o dia a dia do respondente. Objetiva verificar os sintomas de depressão definidos pelo Manual Diagnóstico e Estatístico dos Transtornos Mentais (DSM-V). O PHQ-9 foi o primei- 
ro instrumento desenvolvido especificamente para o rastreamento da depressão nos cuidados primários de saúde e apresentou, no estudo original, excelentes propriedades psicométricas para a identificação da depressão maior, com uma concordância de 93\% em relação às avaliações independentes de profissionais da saúde mental (SPITZER et al., 1999). Estudo indica correlações de rho de 0,387 ( $<<0,000)$, confiabilidade kappa de 0,41 ( $\mathrm{p}<0,001)$, o que indica sua capacidade de rastreio de sintomas depressivos (MATIAS et al., 2016).

Questionário de Informações Pessoais Individual: O questionário buscou coletar dados pessoais dos servidores cuja finalidade foi o de constituir o perfil da amostra estudada, as questões diziam respeito à dimensão sociodemográfica. O instrumento foi desenvolvido pelos pesquisadores.

Os dados foram tratados com o programa livre PSPP - sem significado oficial - versão 0.7.8 para Windows, de maneira descritiva e correlacional, tendo sido adotado o nível de significância de $\mathrm{p}<0,05$. Os escores brutos totais (Escore Total) de todos os instrumentos foram comparados para a validação concorrente da escala PHQ-9.

\section{RESUltados}

Após a descrição dos dados dos participantes (Tabela 1), foram utilizadas estatísticas descritivas e correlacionais inferenciais para o cruzamento das médias entre o PHQ-9 e a HAM-D. A escala em análise foi o PHQ-9 e seus escores, tendo a Escala de Hamilton-D servido de base para atestar a validade, praticidade e coerência da primeira.

Tabela 1 - Características da amostra incluída na validação do Patient Health Questionnaire-9 (PHQ9) $(\mathrm{N}=131)$.

\begin{tabular}{|c|c|c|}
\hline Variáveis & $\operatorname{Amostra}(\mathrm{N})$ & $\%$ \\
\hline \multicolumn{3}{|l|}{ Sexo } \\
\hline Homem & 113 & 86,3 \\
\hline Mulher & 18 & 13,7 \\
\hline \multicolumn{3}{|l|}{ Idade } \\
\hline 18 a 25 & 10 & 7,6 \\
\hline 26 a 30 & 35 & 26,7 \\
\hline 31 a 35 & 53 & 40,5 \\
\hline 36 a 40 & 25 & 19,1 \\
\hline+40 & 8 & 6,1 \\
\hline \multicolumn{3}{|l|}{ Estado civil } \\
\hline Casado & 75 & 57,3 \\
\hline Solteiro & 38 & 29 \\
\hline União estável & 13 & 9,9 \\
\hline Separado & 5 & 3,8 \\
\hline
\end{tabular}

Fonte: Elaborado pelos autores. 
As médias do PHQ-9 e da HAM-D foram comparadas entre si, conforme apresentado na Tabela 2, na tentativa de mostrar a possível equivalência entre as pontuações do PHQ-9 com os níveis de depressão na Hamilton-D.

Tabela 2- Notas de corte do PHQ-9 em relação à HAM-D.

\begin{tabular}{|c|c|c|c|c|c|c|}
\hline $\begin{array}{l}\text { Variáveis } \\
\text { HAM-D }\end{array}$ & $\begin{array}{l}\text { Média } \\
\text { geral }\end{array}$ & $\begin{array}{l}\text { Não } \\
\text { deprimido }\end{array}$ & $\begin{array}{l}\text { Pouco } \\
\text { deprimido }\end{array}$ & $\begin{array}{l}\text { Depressão } \\
\text { moderada }\end{array}$ & $\begin{array}{l}\text { Depressão } \\
\text { grave }\end{array}$ & $\begin{array}{l}\text { Depressão } \\
\text { muito } \\
\text { grave }\end{array}$ \\
\hline Média (PHQ-9) & 4,47 & 2,41 & 4,58 & 7,55 & 11,50 & 10,25 \\
\hline Desvio Padrão & 4,13 & 2,35 & 3,34 & 4,59 & 2,39 & 4,71 \\
\hline Média (HAM-D) & 8,77 & 3,32 & 10,17 & 15,91 & 21,38 & 26,38 \\
\hline Desvio Padrão & 7,30 & 2,23 & 1,81 & 1,58 & 1,30 & 2,00 \\
\hline Total (N) & 131 & 68 & 36 & 11 & 8 & 8 \\
\hline
\end{tabular}

Fonte: Elaborado pelos autores.

Os valores das categorias acima foram rearranjados em subgrupos de três níveis gerais de depressão e verificou-se a um Intervalo de Confiança (IC) de 95\%. Desse modo, conforme mostra a Figura 1, o PHQ-9 foi capaz de alocar os sintomas depressivos em três níveis distintos da Hamilton-D.

Figura 1 - Escores de sintoma depressivos no PHQ-9x níveis de depressão na HAM-D.

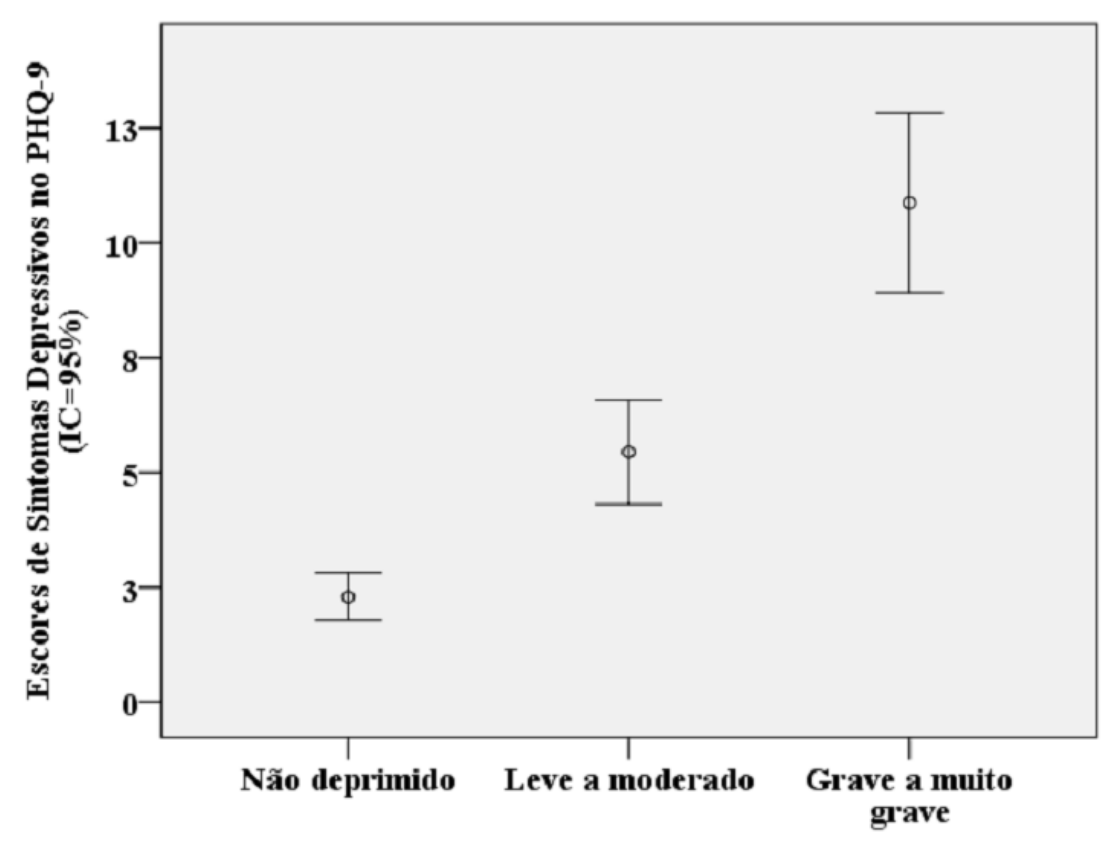

Níveis de Depressão na Escala de Hamilton

Fonte: Elaborado pelos autores. 
Uma vez que o PHQ-9 demonstrou sensibilidade para discriminar os servidores não deprimidos dos servidores com níveis mais brandos e mais graves da sintomatologia, procedeu-se à checagem da capacidade do PHQ-9 identificar casos de ideação suicida ou tentativa de suicídio. Todos os casos de ideação suicida foram identificados conforme os critérios de avaliação da Hamilton-D, tendo sido encontrado que a partir da pontuação igual a 10 no PHQ-9 surgiram todos os casos de ideação suicida identificados pela Hamilton-D. Como não houve identificação de casos de tentativa de suicídio pela Hamilton-D, esse item não pode ser checado. Observa-se, pela Tabela 3, o comparativo entre as médias do PHQ-9 e da Hamilton-D para ideação suicida.

Tabela 3- Nota de corte do Patient Health Questionnaire-9 (PHQ-9) (N = 131) para ideação suicida.

\begin{tabular}{llll}
\hline Sem ideação suicida & N & Média & \multicolumn{2}{c}{ Desvio Padrão } \\
\hline HAM-D & 122 & 7,68 & 6,16 \\
\hline PHQ-9 & 122 & 4,04 & 3,70 \\
\hline Com ideação suicida & & & \\
\hline HAM-D & 9 & 23,56 & 5,07 \\
\hline PHQ-9 & 9 & 10,33 & 5,32 \\
\hline
\end{tabular}

Fonte: Elaborado pelos autores.

Por fim, procedeu à análise da correlação entre os resultados brutos do PHQ-9 com a HAM-D, obtendose pelo teste de Spearman significativa e relativamente forte concordância entre as escalas $(\mathrm{N}=131$; rho $=0,667 ; \mathrm{p}<0,001)$.

\section{Discussão}

A validade de um instrumento psicológico é avaliada pela comparação dos resultados do instrumento com outras medidas consideradas significativas e confiáveis (ERTHAL, 2009). Uma maneira de mensurar a validade critério, adotada nesta pesquisa, é pelo uso da validade concorrente ou simultânea. Trata-se da correlação entre os resultados do instrumento e um critério, que pode ser outro teste psicológico já validado, sem haver um considerável lapso de tempo entre as medidas (ERTHAL, 2009). Se o resultado no primeiro instrumento conseguir discriminar o grupo ao qual um sujeito pertence pelo resultado no segundo teste, diz-se que o instrumento possui boa validade concorrente (BORSA; DAMÁSIO; BANDEIRA, 2012).

Uma descoberta importante da pesquisa foi a identificação do ponto de corte para ideação suicida entre os policiais penais de Porto Velho/RO, no que tange ao índice de sintomas depressivos. Tomando por base a entrevista realizada com os servidores e comparando a mesma com os escores encontrados no PHQ-9, identificou-se o quadro de ideação suicida a partir de 10 pontos, podendo figurar como ponto de corte e um norteador para os casos mais graves de depressão. Esta foi uma inovação deste estudo, uma vez que as pesquisas de validação do PHQ-9 identificam pontuação sensível para o diagnóstico de depressão, sem verificar a capacidade do instrumento para identificar o ponto de corte para rastreio de ideação suicida (ADEWUYA; OLA; AFOLABI, 2006; SZKLO; JAVIER NIETO, 2007; SANTOS et al., 2013; GUERRA et al., 2018).

A prevalência de ideação suicida no presente estudo foi de 6,9\%. Estudo da Organização Mundial de Saúde (2016) aponta os transtornos depressivos como responsáveis por 30\% dos casos de suicídio relatados em todo o mundo. Em relação à prevenção do suicídio, um aspecto a ser discutido é a qualidade dos serviços de saúde e seus profissionais no atendimento a pessoas que tentaram o suicídio, onde não se costuma acompanhar estes pacientes pós-evento, negligenciando a importância vital do encaminhamen- 
to para serviços de atenção em saúde mental para tratamento e orientação dos familiares (BARBOSA; MACEDO; SILVEIRA, 2011). Segundo Botega (2002), entre 15 e 25\% das pessoas que tentam suicídio repetirão a tentativa no ano seguinte, e $10 \%$ efetivamente conseguirão nos próximos 10 anos.

A prevenção do suicídio é de suma importância no enfrentamento da depressão, pois a avaliação dos fatores de risco (impulsividade, agressividade, retraimento, falta de suporte social) e dos fatores de proteção (presença de suporte social, mecanismos de enfrentamento) são essenciais para um melhor manejo do paciente suicida, o que exige uma abordagem ao mesmo tempo diretiva, complexa e multidisciplinar (BARBOSA; MACEDO; SILVEIRA, 2011). Por isso a necessidade de se ter instrumentos confiáveis, com baixo custo e fáceis de manusear visando à identificação de sintomas depressivos.

Na amostra, o questionário PHQ-9 foi capaz de discriminar três grandes estratos de polícia penal, partindo dos não deprimidos até aqueles com depressão grave. Ficando indicado que o PHQ-9 pode atuar muito bem como rastreador geral de sintomas de depressão, apesar de não demonstrar a mesma exatidão para avaliar os níveis mais específicos em particular quando comparado com o HAM-D. No cruzamento entre os escores da Hamilton-D e do PHQ-9, ficou clara a alta sensibilidade entre ambos, com os grupos do PHQ-9 se enquadrando perfeitamente dentro dos escores de depressão da Escala de Hamilton-D, em três níveis de gravidade.

A HAM-D é extremamente eficiente para detectar sintomas de depressão, sendo largamente utilizada no mundo (RIZZOLO, 2008; CALIL; PIRES, 1998), e neste estudo foi usada como padrão ouro, servindo de parâmetro para a análise da eficiência do PHQ-9. Na correlação entre a Escala de Hamilton-D e o PHQ-9, apresentou-se uma magnitude relativamente forte e significativa, o que demonstra que o PHQ-9 pode ser utilizado na atenção primária em substituição a testes mais elaborados, demorados, caros e de complexa execução, sem perder eficiência.

Os achados nesta pesquisa se assemelham ou são mais significativos aos encontrados em estudos que contaram com o mesmo instrumento para obtenção de dados de validação do PHQ-9, é o caso da pesquisa desenvolvida por Guerra et al. (2018) ocorrida entre os anos de 2015 a 2017 com 76 pacientes ambulatoriais acompanhados em uma clínica especializada de insuficiência cardíaca. Nesse estudo os pesquisadores rastrearam sintomas depressivos por meio da Escala de Avaliação de Depressão de Hamilton (HAM-D), pelo Inventário de Depressão de Beck-II (BDI-II) e pelo Patient Health Questionnaire-9 (PHQ-9). Os dados analisados, pelo coeficiente kappa de Fleiss $\left(\mathrm{k}_{\mathrm{F}}\right)$, coeficiente alfa de Krippendorff $\left(\mathrm{C}_{\mathrm{k}}\right)$ e coeficiente alfa de Cronbach, demonstraram haver concordância entre os três instrumentos apenas na ordem de 47,4\% $(\mathrm{n}=36)$. A análise revelou uma concordância superficial $\left(\mathrm{k}_{\mathrm{F}}=\mathrm{C}_{\mathrm{k}}=0,27\right)$ e consistência moderada $(\downarrow \mathrm{C}=$ 0,602 , significativamente não nulo, $p=0,000$ ). Tais resultados afirmam a capacidade de rastreamento de sintomatologia depressiva naquela amostra, porém o PHQ-9 foi nessa pesquisa citada conservador para diagnosticar a depressão.

A pesquisa conduzida por Matias et al. (2016) teve por objetivo determinar a prevalência dos sintomas depressivos entre idosos e correlacionar a concordância entre os métodos de rastreamentos utilizados. No estudo utilizou-se o PHQ-9 e Escala de Depressão Geriátrica de Yesavage-15. Os achados científicos identificaram que a prevalência dos sintomas depressivos rastreados pelo PHQ-9 foi de 62,8\% e, pela Escala de Depressão Geriátrica, foi 52,6\%. A correlação de Spearman entre os resultados das escalas obteve rho $=0,387, \mathrm{p}<0,000$. O coeficiente de confiabilidade de Kappa foi 0,41 e significância de $\mathrm{p}<0,001$. Estes dados permitem afirmar uma moderada concordância estre os instrumentos.

Em outra pesquisa, Adewuya, Ola e Afolabi (2006), com 512 universitários nigerianos, verificaram a validade do PHQ-9 como uma ferramenta de triagem para sintomas de depressão entre estudantes universitários, apresentando boas propriedades psicométricas; entre suas maiores virtudes estavam a sua validade, confiabilidade, brevidade e facilidade de administração. No estudo em questão, o PHQ-9 teve como correlação o inventário de Beck de Depressão (BDI) com uma significativa validade concorrente 
( $r h o=0,67 ; \mathrm{p}<0,001$ ), o que corrobora com os resultados do presente estudo com policiais penais e com a ideia de que o PHQ-9 pode ser uma eficiente ferramenta na atenção primária.

Cabe destacar que normalmente nos estudos relacionados ao uso ou validação do PHQ-9 utilizaram como padrão a aplicação direta do mesmo juntamente com um segundo teste de confirmação e posteriormente compararam resultados brutos buscando-se discriminar cada subgrupo. No presente estudo, diferentemente, optou-se pela administração de uma entrevista diagnóstica da depressão por meio da Hamilton-D como padrão ouro para comparar seus resultados com os valores do PHQ-9, caracterizando uma distinta contribuição à literatura especializada. A escolha da HAM-D deve-se pelo fato abranger um grande número de sintomas depressivos, especialmente as manifestações somáticas e os aspectos cognitivos, bem como sua ampla utilização mundial (CALIL; PIRES, 1998). Além disso, o presente estudo é pioneiro no Brasil ao abordar na metodologia quantitativa o tema da depressão em policiais penais.

Definitivamente este estudo não é exaustivo, pois ele possui limitações e nuances não analisadas. A leitura dos dados deve levar em conta a heterogeneidade do público e o local onde se encontra trabalhando, somados a estes aspectos o tipo de serviço desenvolvido e os riscos inerentes a ele. Devido a limitações de espaço, também não foram levados em conta neste artigo especificidades quanto às diferenças de sexo, idade ou tempo de serviço e como tais aspectos podem afetar os servidores quanto à prevalência dos sintomas depressivos, o que será feito em outra publicação dos mesmos autores com mais detalhes. O presente artigo limitou-se propositadamente a ter como foco a análise do grau de concordância entre o PHQ-9 e a Hamilton-D.

Assim, o uso do PHQ-9 nas instituições governamentais poderia se tornar uma ótima estratégia de rastreamento de sintomas de casos de depressão na atenção primária. Destacam-se, entre as vantagens do PHQ-9, o seu livre acesso e distribuição, a sua base nos critérios do DSM-V para a identificação da depressão, a brevidade e a facilidade de autopreenchimento, indo ao encontro das demandas de atenção à saúde na esfera governamental, onde, em geral, há carência de recursos para ações de prevenção, acolhimento e atendimento dos servidores públicos necessitados. O teste pode ser usado como ferramenta de identificação inicial no momento em que o servidor apresenta sintomas depressivos ou não, e assim possibilitar a proposição de estratégias e protocolos de enfrentamento para tais casos.

\section{ConClusão}

O estudo serviu para demonstrar que o uso do PHQ-9, como rastreador de sintomas de depressão, pode ser extremamente eficiente, tendo o teste apresentado índices significativos, tanto na identificação de casos sintomáticos quanto dos assintomáticos de depressão, situação confirmada pelos escores obtidos junto a HAM-D. O PHQ-9 se destaca entre os demais instrumentos para identificação de depressão como um questionário que pode ser autoaplicado ou aplicado por entrevistadores treinados (ARROL et al., 2010). Seu foco está nos sintomas de depressão definidos pelo Manual Diagnóstico e Estatístico dos Transtornos Mentais (DSM-V) e não é de aplicação exclusiva por psicólogos. Possui apenas nove questões o que é tido como uma vantagem em estudos epidemiológicos em relação aos demais testes do gênero (SANTOS et al., 2013).

O rastreamento dos sintomas de depressão entre servidores públicos da área da segurança penitenciária, assim como no serviço público federal em geral, é necessário e urgente, tendo em vista a gravidade da doença e a sua alta incidência no serviço público (MISSIATTO et al., 2019).

A validação de um instrumento de domínio público e de fácil aplicação como o PHQ-9 pode contribuir para a investigação mais minuciosa da saúde mental de policiais penais e favorecer a elaboração de políticas de saúde mental a estes profissionais da segurança pública que contribuem para o bom funcionamento social. Por fim, constatar a validade do PHQ-9 coopera para que profissionais da saúde tenham a seu alcance um instrumento para rastreamento de sintomas depressivos, bem como diagnóstico e definição 
Souza et al.

de tratamentos adequados.

\section{REFERÊNCIAS}

ADEWUYA, A. O.; OLA, B. A.; AFOLABI, O. O. Validity of the patient health questionnaire (PHQ-9) as a screening tool for depression amongst Nigerian university students. Journal of affective disorders, v. 96, n. 1-2, p. 89-93, 2006.

ARANTES, D. V. Depressão na atenção primária à saúde. Revista Brasileira de Medicina de Família e Comunidade, v. 2, n. 8, p. 261-270, 2007.

AROS, M. S.; YOSHIDA, E. M. P. Estudos da depressão: Instrumentos de avaliação e gênero. Boletim de Psicologia, v. 59, n. 130, p. 61-76, 2009.

ARROL, B.; GOODYEAR-SMITH, F.; CRENGLE, S.; GUNN J.; KERSE, N.; FISHMAN, T.; FALLOON, K.; HATCHER, S . Validation of PHQ-2 and PHQ-9 to screen for major depression in the primary care population. Annals of Family Medicine, v. 8, p. 348-53, 2010.

BARBOSA F. O.; MACEDO, P. C. M.; SILVEIRA, R. M. C. Depressão e o suicido. Revista da SBPH, v. 14, n. 1, p. 233-243, 2011.

BEZERRA, C. M.; ASSIS, S. G.; CONSTANTINO, P. Sofrimento psíquico e estresse no trabalho de polícia penal: uma revisão da literatura. Ciência \& Saúde Coletiva, v. 21, n. 7, p. 2135-2146, 2016.

BORSA, J. C.; DAMÁSIO, B. F.; BANDEIRA, D. R. Adaptação e validação de instrumentos psicológicos entre culturas: algumas considerações. Paidéia, v. 22, n. 53, p. 423-432, 2012.

BOTEGA N. J. Prática Psiquiátrica no Hospital Geral: interconsulta e emergência. Porto Alegre: Artmed, 2002.

CALIL, H. M.; PIRES, M. L. N. Aspectos gerais das escalas de avaliação de depressão. Revista de Psiquiatria Clínica, v. 25, p. 240-244, 1998.

ERTHAL, T. Manual de psicometria. Rio de Janeiro: Editora Jorge, 2009.

FERNANDES, R.C. P; SILVANY, N. A. M.; SENA, G. M.; LEAL, A. S., CARNEIRO, C. A. P.; COSTA, F. P. M. Trabalho e cárcere: um estudo com polícia penal da Região Metropolitana de Salvador, Brasil. Cadernos de Saúde Pública, v. 18, n. 3, 807-816, 2002.

FREIRE, M. A.; FIGUEIREDO, V. L. M.; GOMIDE, A.; JANSEN, K.; SILVA, R. A.; MAGALHÃES, P. V. S.; KAPCZINSKI, F. P. Escala Hamilton: estudo das características psicométricas em uma amostra do sul do Brasil. Jornal Brasileiro de Psiquiatria, v. 63, n. 4, p. 281-289, 2014.

GUERRA, T. R. B.; VENANCIO, I. C. D.; PINHEIRO, D. M. M.; MENDLOWICZ, M. V.; CAVALCANTI, A. C. D.; MESQUITA, E. D. Métodos de Rastreamento da Depressão em Pacientes Ambulatoriais com Insuficiência Cardíaca. International Journal of Cardiovascular Sciences, v. 31, n. 4, p. 414-421, 2018. 
LOSSNITZER, N.; HERZOG, W.; STÖRK, S.; WILD, B.; MÜLLER-TASCH, T.; LEHMKUHL, E.; REGITZ-ZAGROSEK, V.; PANKUWEIT , S.; MAISCH, B.; ERTL, G.; GELBRICH, G.; ANGERMANN, C. E. Incidence rates and predictors of major and minor depression in patients with heart failure. International Journal of Cardiology, v. 167, n. 2, p. 502-507, 2013.

MATIAS, A. G. C.; FONSÊCA, M. A.; GOMES, M. L. F.; MATOS, M. A. A. Indicadores de depressão em idosos e os diferentes métodos de rastreamento. Eistein, v. 14, n. 1, p. 6-11, 2016.

MISSIATTO, L. A. F; FEITOSA, F. B.; RODRÍGUEZ, T. D. M. Correlações entre Assertividade, Condições Laborais, Estresse e Depressão de Servidores do INSS/RO. Psicologia Ciência e Profissão, v. 39, n. e188004, p. 1-12, 2019.

MiSsiATTO, L. A. F; FEITOSA, F. B.; RODRIGUEZ, T. D. M.; MATHIS, M. A. S. Prevalência de depressão em servidores do Instituto Nacional de Seguridade Social. SMAD, Revista. Eletrônica Saúde Mental Álcool Drogas, v. 15, n. 3, p. 1-6, 2019.

MOLINA M. R. A. L.; WIENER, C. D.; BRANCO, J. C.; JANSEN, K.; SOUZA, L. D. M. D.; TOMASI, E.; SILVA, R. A.; PINHEIRO, R. T. Prevalência de depressão em usuários de unidades de atenção primária. Prevalência de depressão em usuários de unidades básicas de saúde. Revista Psiquiatria Clínica, v.39, n. 6, p. 194-197, 2012.

MORAES, P. R. B. de. A identidade e o papel de agentes penitenciários. Tempo Social, v. 25, n. 1, p. 131-147, 2013.

OMS, Organização Mundial de Saúde. The World Health Report. Suicide. Genebra: OMS, 2000. Disponível em: http: //www.scielo.br/scielo.php?script=sci_arttext\&pid=S1414-98932008000400005. Acesso em: 02 jun. 2016.

OPAS, Organização Pan-Americana da Saúde. Folha informativa - Depressão. Disponível em: https:// www.paho.org/bra/index.php?option=com_content\&view=article\&id=5635:folha-informativa-depressao\&Itemid=1095. Acesso em: 08 mai. 2020.

OSÓRIO, F. L.; MENDES, A.V.; CRIPPA, J. A. S.; LOUREIRO, S. R.. Study of the discriminative validity of the PHQ-9 and PHQ-2 in a sample of Brazilian women in the context of primary health care. Perspectives in Psychiatric Care, v. 45, p. 216-227, 2009.

RIZOLLO, R. C. Estudo mostra que Prozac não funciona. Disponível em: http://www.folhadaregiao. com.br/Materia.php?id=88525. Acesso em: 09 mai. 2020.

SANTOS, I. S.; TAVARES, B.; FRANCK, M. T. N.; ALMEIDA, L. S. P.; SILVA, N. T. B.; TAMS, B. D.; PATELLA, A. M.; MATIJASEVICH, A.. Sensibilidade e especificidade do Patient Health Questionnaire-9 (PHQ-9) entre adultos da população geral. Cadernos de Saúde Pública, v. 29, n. 8, p. 1533-1543, 2013.

SPITZER, R. L.; KROENKE, K.; WILLIAMS, J. B. W. (1999). Validation and utility of a self-report version of PRIME-MD: The PHQ primary care study. JAMA, v. 282, p. 1737-1744, 1999. 
SZKLO, M.; JAVIER N. F. Epidemiology: beyond the basics. Sudbury: Jones and Bartlett Publishers, 2007.

WEBB-MURPHY, J. A.; DE LA ROSA, G. M.; SCHIMITZ, K. J.; VISHNYAK, E. J.; RADUCHA, S. C.; ROESCH, S. C.; JOHNSTON, S. L. Operational stress and correlates of mental health among Joint Task Force Guantanamo Bay military personnel. Journal of Traumatic Stress, v. 28, n. 6, p.499-504, 2015

ZIMMERMAN, M. Using the 9-Item Patient Health Questionnaire to Screen for and Monitor Depression. JAMA, v. 322, n. 21, p. 2125-2126, 2019. 